Homology, Homotopy and Applications, vol.5(2), 2003, pp.83-93

\title{
COMPUTATION OF COHOMOLOGY OPERATIONS ON FINITE SIMPLICIAL COMPLEXES
}

\author{
R. GONZÁLEZ-DÍAZ AND P. REAL \\ (communicated by Gunnar Carlsson)
}

\begin{abstract}
We propose a method for calculating cohomology operations on finite simplicial complexes.

Of course, there exist well-known methods for computing (co)homology groups, for example, the "reduction algorithm" consisting in reducing the matrices corresponding to the differential in each dimension to the Smith normal form, from which one can read off the (co)homology groups of the complex [Mun84], or the "incremental algorithm" for computing Betti numbers [DE93]. Nevertheless, little is known about general methods for computing cohomology operations.

For any finite simplicial complex $K$, we give a procedure including the computation of some primary and secondary cohomology operations. This method is based on the transcription of the reduction algorithm mentioned above, in terms of a special type of algebraic homotopy equivalences, called contractions [McL75], of the (co)chain complex of $K$ to a "minimal" (co)chain complex $M(K)$. More concretely, whenever the ground ring is a field or the (co)homology of $K$ is free, then $M(K)$ is isomorphic to the (co)homology of $K$. Combining this contraction with the combinatorial formulae for Steenrod reduced $p$ th powers at cochain level developed in [GR99] and [Gon00], these operations at cohomology level can be computed. Finally, a method for calculating Adem secondary cohomology operations $\Phi_{q}: \operatorname{Ker}\left(S q^{2} H^{q}(K)\right) \rightarrow$ $H^{q+3}(K) / S q^{2} H^{q}(K)$ is showed.
\end{abstract}

\section{Introduction}

Particular important topological invariants are the (co)homology groups. In a certain way, these groups measure the degree of connectedness of the space. Al-

Authors are partially supported by the PAICYT research project FQM-296 from Junta de Andalucia and the DGES-SEUID research project PB98-1621-C02-02 from Education and Science Ministry (Spain).

Received August 14, 2001, revised July 17, 2002; published on April 22, 2003.

2000 Mathematics Subject Classification: 55S05, 55U10.

Key words and phrases: Cohomology operations, finite simplicial complexes.

(C) 2003, R. González-Díaz and P. Real. Permission to copy for private use granted. 
though there are plenty of programs for calculating (co)homology groups of finite simplicial complexes, we have not found any general software for computing cohomology operations.

Our main motivation is the design of a program for computing all sort of cohomology invariants on finite simplicial complexes: (co)homology groups, cup product, Bockstein cohomology operation, cohomology operations determined by homomorphisms of coefficient groups, Steenrod squares and reduced $p$ th powers, Pontrjagin squares and $p$ th powers, the $A_{\infty}$-algebra structure of cohomology, higher cohomology operations, etc. In this paper, we give a solution to the problem of computing Steenrod squares and reduced $p$ th powers $[$ Ste47, ES62] and Adem secondary cohomology operations [Ade52, Ade58]. Our approach is based on two facts. First, the translation of the well-known "reduction" algorithm for computing (co)homology groups [Mun84] in terms of homotopy equivalences. In that way, we have a description of the generators of the (co)homology in terms of (co)chains. Second, the combinatorial formulae for the cohomology operations mentioned above stablished in [GR99] and [Gon00]. Using the same approach we think that the rest of cohomology operations could be attacked.

\section{Background}

We give a brief summary of concepts and notation used in the following sections. Our terminology follows Munkres [Mun84] and McLane [McL75].

For $0 \leqslant q \leqslant n$, a $q$-simplex $\sigma$ in $\mathbf{R}^{n}$ is the convex hull of a set $T$ of $q+1$ affinely independent points $\left(v_{0}, \ldots, v_{q}\right)$. The dimension of $\sigma$ is $|\sigma|=q$. For every non-empty $U \subset T$, the simplex $\tau$ defined by $U$ is a face of $\sigma$. A simplicial complex $K$ is a collection of simplices satisfying the following properties:

- If $\tau$ is a face of $\sigma$ and $\sigma \in K$ then $\tau \in K$.

- If $\sigma, \tau \in K$ then $\sigma \cap \tau$ is either empty or a face of both.

The set of all the $q$-simplices of $K$ is denoted by $K^{(q)}$. The largest dimension of any simplex in $K$ is the dimension of $K$. A simplex $\sigma$ in $K$ is maximal if it is not face of any simplex in $K$. Therefore, $K$ can be given by the set of its maximal simplices. A subset $L \subset K$ is a subcomplex of $K$ if it is a simplicial complex itself. All simplices in this paper have finite dimension and all simplicial complexes are finite collections. From now on, $K$ denotes a finite simplicial complex. The oriented $q-$ simplex $\sigma=\left[v_{0}, \ldots, v_{q}\right]$ is the equivalence class of the particular ordering $\left(v_{0}, \ldots, v_{q}\right)$. Two orderings are equivalent if they differ from one another by an even permutation.

Let $\Lambda$ denote an abelian group. A formal sum, $\lambda_{1} \sigma_{1}+\cdots+\lambda_{n} \sigma_{n}$, where $\lambda_{i} \in \Lambda$ and $\sigma_{i}$ are oriented $q$-simplices, is called a $q$-chain. The chain complex canonically associated to $K$, denoted by $C_{*}(K)$, is the family of groups such that in each dimension $q, C_{q}(K)$ is the group of $q$-chains in $K$. The boundary of a $q$-simplex $\sigma=\left[v_{0}, \ldots, v_{q}\right]$ is the $(q-1)$-chain

$$
\partial_{q} \sigma=\sum_{i=0}^{q}(-1)^{i}\left[v_{0}, \ldots, \hat{v}_{i}, \ldots, v_{q}\right]
$$


where the hat means that $v_{i}$ is omitted. By linearity, the boundary operator $\partial_{q}$ can be extended to $q$-chains, where it is a homomorphism. It is clear that for each $q$-simplex $\sigma_{j}$ there exist unique integers $\lambda_{i j}$ such that

$$
\partial_{q}\left(\sigma_{j}\right)=\sum_{\tau_{i} \in K^{(q-1)}} \lambda_{i j} \tau_{i}
$$

The matrix $A_{q}=\left(\lambda_{i j}\right)$ is the matrix of $\partial_{q}$ relative to the basis $K^{(q)}$ and $K^{(q-1)}$. The group of $q$-cycles, $Z_{q}(K)$, is the kernel of $\partial_{q}$, and define $Z_{0}(K)=C_{0}(K)$. The group of $q$-boundaries, $B_{q}(K)$, is the image of $\partial_{q+1}$, that is, the subgroup of $q$-chains $b \in C_{q}(K)$ for which there exists a $(q+1)$-chain $a$ with $b=\partial_{q+1} a$. It can be shown that $\partial_{q} \partial_{q+1}$ is null so $B_{q}(K)$ is a subgroup of $Z_{q}(K)$. Then, the $q$ th homology group

$$
H_{q}(K)=Z_{q}(K) / B_{q}(K)
$$

can be defined for each integer $q$. Given that elements of this group are cosets of the form $a+B_{q}(K)$, where $a \in Z_{q}(K)$, we say that the coset $a+B_{q}(K)$, denoted by $[a]$, is the homology class in $H_{q}(K)$ determined by $a$ or $a$ is a representative cycle of $[a]$.

Let $K$ and $L$ be two simplicial complex. A chain map $f: C_{*}(K) \rightarrow C_{*}(L)$ is a family of homomorphisms

$$
\left\{f_{q}: C_{q}(K) \rightarrow C_{q}(L)\right\}_{q \geqslant 0}
$$

such that $\partial_{q} f_{q}=f_{q-1} \partial_{q}$, for all $q$.

Dual concepts to the previous ones can be defined. The cochain complex canonically associated to $K, C^{*}(K)$, is the family

$$
C^{*}(K)=\left\{C^{q}(K), \delta_{q}\right\}_{q \geqslant 0},
$$

where

$$
C^{q}(K)=\operatorname{Hom}\left(C_{q}(K) ; \Lambda\right)=\left\{c: C_{q}(K) \rightarrow \Lambda, \quad c \text { is a homomorphism }\right\}
$$

and

$$
\delta^{q}: C^{q}(K) \rightarrow C^{q+1}(K)
$$

called the coboundary operator is given by

$$
\delta^{q}(c)(a)=c\left(\partial_{q+1} a\right),
$$

where $c \in C^{q}(K)$ and $a \in C_{q+1}(K)$. Observe that a $q$-cochain can be defined only on $K^{(q)}$ and extended to $C_{q}(K)$ by linearity. Moreover, if $\Lambda$ is a ring, then a base of $C^{q}(K)$ is the set of homomorphisms

$$
\sigma^{*}: C_{q}(K) \rightarrow \Lambda
$$


such that if $\tau \in K^{(q)}$, then $\sigma^{*}(\tau)=1$ if $\tau=\sigma$, and $\sigma^{*}(\tau)=0$ otherwise. $Z^{q}(K)$ and $B^{q}(K)$ are the kernel of $\delta^{q}$ and the image of $\delta^{q-1}$, respectively. The elements in $Z^{q}(K)$ are called $q$-cocycles and those in $B^{q}(K)$ are called $q$-coboundaries. It is also satisfied that $\delta^{q} \delta^{q-1}=0$ so the $q$ th cohomology group

$$
H^{q}(K)=Z^{q}(K) / B^{q}(K)
$$

can also be defined for each integer $q$. If $\Lambda$ is a ring, the cohomology of $K$ is also a ring with the cup product

$$
\smile: H^{p}(K) \otimes H^{q}(K) \rightarrow H^{p+q}(K)
$$

defined as follows. Let $[c] \in H^{p}(K)$ and $\left[c^{\prime}\right] \in H^{q}(K)$. Then $[c] \smile\left[c^{\prime}\right]$ is the class of cohomology determined by the cocycle $c \smile c^{\prime}$, defined by

$$
c \smile c^{\prime}\left[v_{0}, \ldots, v_{p+q}\right]=\mu\left(c\left[v_{0}, \ldots, v_{p}\right] \otimes c^{\prime}\left[v_{p}, \ldots, v_{p+q}\right]\right),
$$

where $v_{0}<\cdots<v_{p+q}$ and $\mu$ is the product on $\Lambda$.

We use in this paper a special type of homotopy equivalences: contractions [McL75]. A contraction $r$ of a chain complex $N_{*}$ to another chain complex $M_{*}$ is a set of three homomorphisms $(f, g, \phi)$ where $f: N_{n} \rightarrow M_{n}$ (projection) and $g$ : $M_{n} \rightarrow N_{n}$ (inclusion) are chain maps and satisfy that $f g=1_{M}$, and $\phi: N_{n} \rightarrow N_{n+1}$ (homotopy operator) satisfies that

$$
1_{N}-g f=\phi \partial_{N}+\partial_{N} \phi .
$$

Moreover, $\phi g=0, f \phi=0$, and $\phi \phi=0$. In this definition we follow EilenbergMacLane terminology [EM52]; we also find in the literature "strong deformation retraction" or SDR (Lambe-Stasheff [LS87], Gugenheim-Lambe [GL89] and Gugenheim-Stasheff [GS86]), "Eilenberg-Zilber data" (Gugenheim-Munkholm [GM74]), or "trivial extension" (Munkholm [Mun76]). Since the emergence of the Homological Perturbation Theory [GL89, GLS91, HK91], contractions have been frequently used. We can cite, for example, [Hue86, LS87, Ser94, Rea00].

A contraction up to dimension $n$ of $N_{*}$ to $M_{*}$ consists in a set of three homomorphisms $(f, g, \phi)$ such that

$$
f_{k}: N_{k} \rightarrow M_{k}, \quad g_{k}: M_{k} \rightarrow N_{k} \quad \text { and } \quad \phi_{k-1}: N_{k-1} \rightarrow N_{k}
$$

are defined for all $k \leqslant n, \phi_{n}=0$, and the conditions of being a contraction are satisfied up to dimension $n$.

Given a contraction $r=(f, g, \phi)$ of $N_{*}$ to $M_{*}$, it is possible to obtain another contraction $r^{*}=\left(f^{*}, g^{*}, \phi^{*}\right)$ of $\operatorname{Hom}(N ; \Lambda)$ to $\operatorname{Hom}(M ; \Lambda)$ as follows:

$$
\begin{gathered}
f^{*}: \operatorname{Hom}\left(N_{n} ; \Lambda\right) \rightarrow \operatorname{Hom}\left(M_{n} ; \Lambda\right), \quad g^{*}: \operatorname{Hom}\left(M_{n} ; \Lambda\right) \rightarrow \operatorname{Hom}\left(N_{n} ; \Lambda\right), \\
\phi^{*}: \operatorname{Hom}\left(N_{n} ; \Lambda\right) \rightarrow \operatorname{Hom}\left(N_{n-1} ; \Lambda\right),
\end{gathered}
$$


are such that

$$
f^{*}(c)=c g, \quad g^{*}\left(c^{\prime}\right)=c^{\prime} f \quad \text { and } \quad \phi^{*}(c)=c \phi,
$$

where $c \in \operatorname{Hom}\left(N_{n} ; \Lambda\right)$ and $c^{\prime} \in \operatorname{Hom}\left(M_{n} ; \Lambda\right)$.

\section{3. "Minimal" Chain Complexes}

It is possible to translate the results of the "reduction algorithm", discussed at length in [Mun84], in terms of contractions. Combining this translation with modern homological perturbation techniques, algorithms for computing algebraic invariants, such as the cohomology of $K$ and primary and secondary cohomology operations can be designed in an easy way.

First of all, it is necessary to recall the reduction algorithm for computing homology groups of a finite simplicial complex $K$. This method consists in reducing the matrix $A$ of the boundary operator in each dimension $q$, relative to given basis of $C_{q}(K)$ and $C_{q-1}(K)$, to its Smith normal form $A^{\prime}$ (a matrix of integers satisfying that all its elements are zero except for $\lambda_{11}^{\prime} \geqslant 1$ and $\lambda_{11}^{\prime}\left|\lambda_{22}^{\prime}\right| \cdots \mid \lambda_{\ell \ell}^{\prime}$ for some integer $\ell$, where $\mid$ means "is a factor of"). This reduction is done in each dimension $q$ modifying the given base of $C_{q-1}(K)$, using the following "elementary row operations" on the matrix $A$ :

(1) Exchange row $i$ by row $k$.

(2) Multiply row $i$ by -1 .

(3) Replace row $i$ by row $i+n$ (row $k$ ), where $n$ is an integer and $k \neq i$.

Of course, there are similar "column operations" on $A$ corresponding to changes of basis of $C_{q}(K)$. With this operations, the Smith normal form $A^{\prime}$ of $A$ can be obtained, relative to some basis $\left\{a_{1}, \ldots a_{r}\right\}$ of $C_{q}(K)$ and $\left\{e_{1}, \ldots, e_{s}\right\}$ of $C_{q-1}(K)$. Then,

(1) $\left\{a_{\ell+1}, \ldots, a_{r}\right\}$ is a base of $Z_{q}(K)$,

(2) $\left\{\lambda_{11}^{\prime} e_{1}, \ldots, \lambda_{\ell \ell}^{\prime} e_{\ell}\right\}$ is a base of $B_{q-1}(K)$.

Obviously, a dual treatment for $C^{*}(K)$ and, consequently, for the cohomology of $K$, can be done.

A chain complex $M_{*}(K)$ is called minimal if in each dimension $q, M_{q}(K)$ is a finitely generated free abelian group and the Smith normal form $A^{\prime}$ of the differential of $M_{q}(K)$ has the first element $\lambda_{11}^{\prime}$ different from 1 . An algebraic minimal model of $K$ is a minimal chain complex $M_{*}(K)$ together with a contraction of $C_{*}(K)$ to $M_{*}(K)$. Indeed, there is an algebraic minimal model for any finite simplicial complex $K$ and any two algebraic minimal models of $K$ are isomorphic. The concept of algebraic minimal model is old (e.g. [Tat57]).

Now, let us construct inductively an algebraic minimal model of a given finite simplicial complex $K$. Suppose that an algebraic minimal model up to dimension $q-1$ is already constructed. That is, we have a minimal chain complex $M_{*}^{\prime}(K)$ such that $M_{i}^{\prime}(K)=0, i \geqslant q$, and a contraction up to dimension $q-1,\left(f^{\prime}, g^{\prime}, \phi^{\prime}\right)$, of $C_{*}(K)$ to $M_{*}^{\prime}(K)$. Reduce the matrix of $\partial_{q}: C_{q}(K) \rightarrow C_{q-1}(K)$ to its Smith 
normal form $A^{\prime}$. If the elements $\lambda_{11}^{\prime}=\cdots=\lambda_{t t}^{\prime}=1$, for $t \leqslant \ell$ (that is, $\partial\left(a_{i}\right)=e_{i}$ for $1 \leqslant i \leqslant t$ ), then define $M_{*}(K)$ as follows:

$$
\begin{aligned}
& M_{i}(K)=M_{i}^{\prime}(K), \quad \text { for } i \neq q-1, q \\
& M_{q-1}(K)=M_{q-1}^{\prime}(K)-\Lambda\left[e_{1}, \ldots, e_{t}\right] \\
& M_{q}(K)=C_{q}(K)-\Lambda\left[a_{1}, \ldots, a_{t}\right]
\end{aligned}
$$

where $\Lambda\left[a_{1}, \ldots, a_{t}\right]$ and $\Lambda\left[e_{1}, \ldots, e_{t}\right]$ are the free abelian groups generated by $\left\{a_{1}, \ldots, a_{t}\right\}$ and $\left\{e_{1}, \ldots, e_{t}\right\}$, respectively. The formulae for the component morphisms of the contraction up to dimension $q,(f, g, \phi)$, of $C_{*}(K)$ to $M_{*}(K)$ are:

$$
\begin{aligned}
& f(x)= \begin{cases}f^{\prime}(x) & \text { if } x \in \Lambda\left[e_{t+1}, \ldots, e_{s}\right] \text { or } x \in C_{i}(K), i<q, \\
0 & \text { if } x \in \Lambda\left[e_{1}, \ldots, e_{t}\right] \text { or } x \in \Lambda\left[a_{1}, \ldots, a_{t}\right], \\
x & \text { if } x \in \Lambda\left[a_{t+1}, \ldots, a_{r}\right],\end{cases} \\
& g(y)= \begin{cases}g^{\prime}(y) & \text { if } y \in M_{i}(K), i<q, \\
y & \text { if } y \in M_{q}(K),\end{cases} \\
& \phi(x)=\phi^{\prime}(x) \quad \text { if } x \in C_{i}(K), i<q-1, \\
& \phi\left(e_{i}\right)=a_{i} \quad \text { if } 1 \leqslant i \leqslant t, \\
& \phi\left(e_{i}\right)=0 \quad \text { if } t+1 \leqslant i \leqslant s .
\end{aligned}
$$

In this way, we can determine an algebraic minimal model for a finite simplicial complex $K$. Observe that whenever $\Lambda$ is a field or the homology of $K$ is free, then $M_{*}(K)$ is isomorphic to $H_{*}(K)$ and, therefore, we can obtain a contraction of $C_{*}(K)$ to its homology.

Passing to cohomology does not represent a problem and a dual process can be done without effort. More concretely, take into account that if the ground ring $\Lambda$ is a field then, the homology and cohomology of $K$ are isomorphic. Moreover, given a generator of homology, $\alpha$, of degree $q$, we can define the corresponding generator of cohomology $\alpha^{*}: H_{q}(K) \rightarrow \Lambda$ as

$$
\alpha^{*}(\alpha)=1 \quad \text { and } \quad \alpha^{*}(\beta)=0 \quad \text { for } \alpha \neq \beta \in H_{q}(K) .
$$

Starting from a contraction $(f, g, \phi)$ from $C_{*}(K)$ to $H_{*}(K)$, we construct a cochain contraction $\left(f^{*}, g^{*}, \phi^{*}\right)$ from $C^{*}(K)$ to $H^{*}(K)$ as follows. Let $c \in C^{q}(K)$ and $\alpha^{*} \in H^{q}(K)$. Define $f^{*} c=c g, g^{*} \alpha^{*}=\alpha^{*} f$ and $\phi^{*} c=c \phi$. Then, for example, we can compute the cohomology ring of $K$ in the following way:

Procedure 1. Algorithm for computing cup product

Take $\alpha^{*} \in H^{p}(K)$ and $\beta^{*} \in H^{q}(K)$

For every $(p+q)$-homology class $\gamma$

compute $\left(g^{*} \alpha^{*}\right) \smile\left(g^{*} \beta^{*}\right)(g \gamma)$

End for 
Notice that the resulting cohomology class is determined by the cocycle $c=$ $\left(g^{*} \alpha^{*}\right) \smile\left(g^{*} \beta^{*}\right)$. We compute this cocycle using the expression (1).

\section{Steenrod Cohomology Operations}

We will see in this section that the homotopy equivalence data obtained in the previous section connecting a chain complex with its homology, is essential for us for computing cohomology operations. Taking a simplicial version $O: C^{*}(K) \rightarrow$ $C^{*+i}(K)$ of a cohomology operation $\mathcal{O}: H^{*}(K) \rightarrow H^{*+i}(K)$ (where $\mathcal{O}$ si defined as follows: let $[c] \in H^{q}(K)$, then $\left.\mathcal{O}[c]:=[O(c)]\right)$ and a contraction $\left(f^{*}, g^{*}, \phi^{*}\right)$ from $C^{*} K$ to $H^{*} K$, it is possible to derive the following algorithm.

Procedure 2. Algorithm for computing cohomology operations.

Take $\alpha^{*}$, a cohomology class of degree $q$;

compute $f^{*}\left(O\left(g^{*} \alpha^{*}\right)\right)$.

Observe that the fundamental data we need are explicit expressions at cochain level for the cohomology operations we want to compute. Moreover, note that the complexity of this algorithm principally depends on the complexity of the explicit expressions of the cohomology operation $\mathcal{O}$ at cocyclic level.

Now, let us suppose $\mathbf{Z}_{p}$ ( $p$ being a prime) is the ground ring and let $(f, g, \phi)$ a contraction that connects $C_{*}(K)$ with its homology. From this datum and the combinatorial formulae for Steenrod squares and reduced $p$ th powers [Ste47, ES62] at cochain level established in [GR99, Gon00], Steenrod cohomology operations $\mathcal{P}_{i}: H^{*}(K) \rightarrow H^{* p-i}(K)$ can effectively be computed. Let us denote the simplicial expression of $\mathcal{P}_{i}$ by $P_{i}$. Therefore, for calculating the cohomology class $\mathcal{P}_{i}\left(\alpha^{*}\right)$ with $\alpha^{*} \in H^{q}(K)$, we only need to compute $P_{i}\left(\alpha^{*} f\right) g$. For instance, the formula for the Steenrod reduced power

$$
\mathcal{P}_{1}: H^{*}(K) \rightarrow H^{* p-1}(K)
$$

at cochain level [Gon00] is:

$$
\begin{aligned}
& P_{1}(c)(\sigma)=\sum_{\substack{1 \leqslant j \leqslant p-1 \\
j q \leqslant i \leqslant(j+1) q-1}}(-1)^{(i+1)(q+1)+1} \\
& \mu\left(c\left[v_{0}, \ldots, v_{q}\right] \otimes c\left[v_{q}, \ldots, v_{2 q}\right] \otimes \ldots\right. \\
& \cdots \otimes c\left[v_{(j-2) q}, \ldots, v_{(j-1) q}\right] \\
& \otimes c\left[v_{(j-1) q}, \ldots, v_{i-q}, v_{i}, \ldots, v_{(j+1) q-1}\right] \\
& \otimes c\left[v_{(j+1) q-1}, \ldots, v_{(j+2) q-1}\right] \otimes \cdots \\
& \cdots \otimes c\left[v_{(p-2) q-1}, \ldots, v_{(p-1) q-1}\right] \\
&\left.\otimes c\left[v_{(p-1) q-1}, \ldots, v_{p q-1}\right] \otimes c\left[v_{i-q}, \ldots, v_{i}\right]\right)
\end{aligned}
$$


where $c$ is a $q$-cocycle, $\sigma=\left[v_{0}, \ldots, v_{p q-1}\right]$ is a $(p q-1)$-simplex such that $v_{0}<\cdots<$ $v_{p q-1}$ and $\mu$ is the product on $\mathbf{Z}_{p}$. Let us notice that the number of summands of $P_{1}$ over a $q$-cocycle $c$ and a $(p q-1)$-simplex $\sigma$ is $(p-1) q$.

The particular case of Steenrod squares $\left(\mathbf{Z}_{2}\right.$ being the ground ring),

$$
S q^{i}: H^{*}(K) \rightarrow H^{*+i}(K),
$$

can be expressed in a matrix form due to the fact that these cohomology operations are homomorphisms. Moreover, the process of diagonalization of such matrices can give us detailed information about the kernel and image of these cohomology operations. We will use this fact in the following section.

\section{Adem Secondary Cohomology Operations}

For attacking the computation of secondary cohomology operations, we will see that the homotopy operator $\phi$ of the contraction $(f, g, \phi)$ from $C_{*}(K)$ to its homology, is essential. Notice that since $\mathbf{Z}_{2}$ is the ground ring, we can compute a cochain contraction $\left(f^{*}, g^{*}, \phi^{*}\right)$ from $C^{*} K$ to $H^{*} K$ as explained before.

First of all, we shall indicate how Adem secondary cohomology operations

$$
\Psi_{q}: N^{q}(K) \rightarrow H^{q+3}\left(K ; \mathbf{Z}_{2}\right) / S q^{2} H^{q+1}(K ; \mathbf{Z}), \quad q \geqslant 2
$$

can be constructed (see $[\mathbf{A d e 5 2}]) . N^{q}(K)$ denotes the kernel of $S q^{2}: H^{q}(K ; \mathbf{Z}) \rightarrow$ $H^{q+2}\left(K ; \mathbf{Z}_{2}\right)$ These operations appear using the known relation:

$$
S q^{2} S q^{2} \alpha+S q^{3} S q^{1} \alpha=0
$$

for any $\alpha \in H^{*}(K ; \mathbf{Z})$. For this particular relation there exist cochain mappings

$$
E_{j}: C^{*}(K \times K \times K \times K) \rightarrow C^{*-j}(K)
$$

such that mod 2

$$
\left(c \smile_{q-2} c\right) \smile_{q}\left(c \smile_{q-2} c\right)+\left(c \smile_{q-1} c\right) \smile_{q-2}\left(c \smile_{q-1} c\right)=\delta E_{3 q-3}\left(c^{4}\right),
$$

where $\smile_{k}$ is the cup $-k$ product [Ste47] and $c$ is a $q-$ cochain. Recall that, at cochain level, $S q^{i}(c)=c \smile_{j-i} c$ mod 2, where $c$ is a $j$-cocycle. Then $\Psi_{q}$ is defined at cochain level by

$$
\psi_{q}(c)=b \smile_{q-1} b+b \smile_{q} \delta b+E_{3 q-3}\left(c^{4}\right)+\eta(c) \smile_{q-3} \eta(c)+\eta(c) \smile_{q-2} \delta \eta(c),
$$

where $c$ is a representative $q$-cocycle of a cohomology class of $N^{q}(K), b$ is a $(q+1)$ cochain such that $c \smile_{q-2} c=\delta b$ and $\eta(c)=\frac{1}{2}\left(c \smile_{q} c+c\right)$.

Since formulae for computing cup $-i$ products are well-known [Ste47], the steps for computing $\Psi_{q}$ are the following:

Procedure 3. Algorithm for computing Adem secondary cohomology operations. 


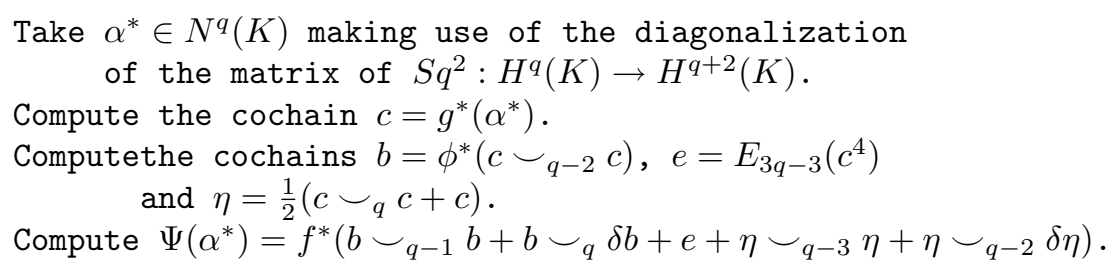

Note that it is easy to prove that

$$
c \smile_{q-2} c=\delta\left(\phi^{*}\left(c \smile_{q-2} c\right)\right),
$$

using the relation $1-g^{*} f^{*}=\phi^{*} \delta+\delta \phi^{*}$.

Let us observe that in order to compute Adem secondary cohomology operations, it is essential for us to have a combinatorial picture of them. A method for obtaining "economical" combinatorial formulae for $E_{3 q-3}$ is given in [Gon00]. For example,

$$
\begin{aligned}
E_{3}\left(c^{4}\right)(\sigma)= & \mu\left(c\left[v_{0}, v_{2}, v_{3}\right] \otimes c\left[v_{0}, v_{1}, v_{2}\right] \otimes c\left[v_{3}, v_{4}, v_{5}\right] \otimes c\left[v_{2}, v_{3}, v_{5}\right]\right. \\
& +c\left[v_{0}, v_{4}, v_{5}\right] \otimes c\left[v_{3}, v_{4}, v_{5}\right] \otimes c\left[v_{0}, v_{1}, v_{2}\right] \otimes c\left[v_{0}, v_{1}, v_{2}\right] \\
& +c\left[v_{0}, v_{1}, v_{5}\right] \otimes c\left[v_{3}, v_{4}, v_{5}\right] \otimes c\left[v_{1}, v_{2}, v_{3}\right] \otimes c\left[v_{1}, v_{2}, v_{3}\right] \\
& +c\left[v_{0}, v_{1}, v_{2}\right] \otimes c\left[v_{2}, v_{4}, v_{5}\right] \otimes c\left[v_{2}, v_{3}, v_{4}\right] \otimes c\left[v_{2}, v_{3}, v_{4}\right] \\
& \left.+c\left[v_{0}, v_{1}, v_{2}\right] \otimes c\left[v_{2}, v_{3}, v_{5}\right] \otimes c\left[v_{3}, v_{4}, v_{5}\right] \otimes c\left[v_{3}, v_{4}, v_{5}\right]\right),
\end{aligned}
$$

where $c$ is a 2-cochain, $\sigma=\left[v_{0}, v_{1}, v_{2}, v_{3}, v_{4}, v_{5}\right]$ is a 5-simplex such that $v_{0}<v_{1}<$ $v_{2}<v_{3}<v_{4}<v_{5}$ and $\mu$ is the product on $\mathbf{Z}_{2}$.

\section{Some Comments}

All these results can be given in a more general framework working with not necessarily finite simplicial complexes. Nevertheless, a contraction of the chain complex associated to the simplicial complex to its (co)homology must exist in order to develop the method.

Concerning the complexity, obtaining a contraction of a finite simplicial complex $K$ to its (co)homology can be done using an "incremental algorithm" given in [ELZ00] which runs in time as most cubic in the number of simplices of the complex if the group of coefficients is a field. Moreover, another study of the computation of a contraction from a chain complex to its homology is done in [KMS98]. In our case, the complexity of our procedure for computing a cohomology operation $\mathcal{O}$, essentially depends on the complexity of the combinatorial expression of $\mathcal{O}$ at cocycle level. Finally, in order to obtain the image of any cohomology operation at cochain level over a representative cocycle using our formulae, we have to compute them over a base of $C_{*}(K)$ in the desired dimension. A way of decreasing the complexity of this is to do a "topological" thinning of the simplicial complex $K$ in order to obtain a thinned simplicial subcomplex $M_{\text {top }}(K)$ of $K$, such that there exists a contraction of $C_{*}(K)$ to $C_{*}\left(M_{\mathrm{top}}(K)\right)$. For example, one way to construct it is using simplicial collapses [For99]. Then, we can apply our machinery for computing 
cohomology operations in the thinned simplicial complex $M_{\text {top }}(K)$ and the results can be easily interpreted in the "big" simplicial complex $K$.

\section{References}

[Ade52] J. Adem. The Iteration of the Steenrod Squares in Algebraic Topology. Proc. Nat. Acad. Sci. USA, v. 38 (1952) 720-724.

[Ade58] J. Adem. Operaciones Cohomológicas de Segundo Orden Asociadas a Cuadrados de Steenrod. Symp. Internacional de Topología Algebraica, Univ. of Mexico, Mexico D.F. (1958) 186-221.

[DE93] C.J.A. Delfinado, H. Edelsbrunner. An Incremental Algorithm for Betti Numbers of Simplicial Complexes. Proc. 9th Ann. Symp. Comput. Geom. (1993) 232-239.

[ELZ00] H. Edelsbrunner, D. Letscher, A. Zomorodian. Topological Persistence and Simplification. Proc. 41st Ann. IEEE Sympos. Found. Comput. Sci. (2000), 454-463.

[EM52] S. Eilenberg, S. MacLane. On the Groups of $H(\Pi, n)$, I. Ann. of Math., v. 58, n. 1 (1952), 55-107.

[ES62] N.E. Steenrod, D.B.A. Epstein. Cohomology Operations. Ann. of Math. Studies, v. 50, Princeton University Press, 1962.

[For99] R. Forman. Combinatorial Differential Topology and Geometry. New Perspective in Geometric Combinatorics. MSRI Publications, v. 8 (1999) 177-206.

[Gon00] R. González-Díaz. Cohomology Operations: A Combinatorial Approach. Ph. D. Thesis, Seville University, May 2000.

[GR99] R. González-Díaz, P. Real. A Combinatorial Method for Computing Steenrod Squares. J. of Pure and App. Alg., v. 139 (1999) 89-108.

[GL89] V.K.A.M. Gugenheim, L. Lambe. Perturbation Theory in Differential Homological Algebra, I. Illinois J. Math., 33 (1989) 556-582.

[GLS91] V.K.A.M. Gugenheim, L. Lambe, J.D. Staheff. Perturbation Theory in Differential Homological Algebra, II. Illinois J. Math., v. 35, n. 3 (1991) 357-373.

[GM74] V.K.A.M. Gugenheim, H.J. Munkholm. On the Extended Functoriality of Tor and Cotor. J. Pure Appl. Algebra, v. 4 (1974) 9-29.

[GS86] V.K.A.M. Gugenheim, J. Stasheff. On Perturbations and $A_{\infty^{-}}$ Structures. Bull. Soc. Math. Belg., v. 38 (1986), 237-246.

[Hue86] J. Huebschmann. The Homotopy Type of $F \Phi^{q}$. The Complex and Symplectic Cases. App. of Algebraic K-Theory to Algebraic Geometry and Number Theory, II. Proc., Boulder, Colorado, 1983. Cont. Math. v. 55 (1986) 487-518.

[HK91] J. Huebschmann, T. Kadeishvili. Small Models for Chain Algebras. Math. Z., v. 207 (1991) 245-280. 
[KMS98] T. Kaczynski, M. Mrozek, M. Slusarek. Homology Computation by Reduction of Chain Complexes. Computers and Mathematics, v. 35, n. 4 (1998) 59-70.

[LS87] L. Lambe, J.D. Stasheff. Applications of Perturbation Theory to Iterated Fibrations. Manuscripta Math., v. 58 (1987) 363-376.

[McL75] S. MacLane. Homology. Classics in Mathematics, Springer-Verlag, Berlin, 1995. Reprint of the 1975 ed.

[Mun76] H.J. Munkholm. The Eilenberg-Moore Spectral Sequence and Strongly Homotopy Multiplicative Maps. J. Pure Appl. Alg., v. 9 (1976) 1-50.

[Mun84] J.R. Munkres. Elements of Algebraic Topology. Addison-Wesley Co., 1984.

[Rea00] P. Real. Homological Perturbation Theory and Associativity. Homology, Homotopy and its Applications, v. 2, n. 5 (2000) 51-88.

[Ser94] F. Sergeraert. The Computability Problem in Algebraic Topology. Adv. Math., v. 104, n. 1 (1994) 1-29.

[Ste47] N.E. Steenrod. Products of Cocycles and Exensions of Mappings. Ann. of Math., v. 48 (1947) 290-320.

[Tat57] J. Tate. Homology of Noetherian Rings and Locla Rings. Ill. J. of Math., v. 1 (1957) 14-27.

This article may be accessed via WWW at http://www.rmi.acnet.ge/hha/ or by anonymous ftp at

ftp://ftp.rmi.acnet.ge/pub/hha/volumes/2003/n2a4/v5n2a4.(dvi,ps,pdf)

R. González-Díaz rogodi@us.es

Dept. of Applied Math., University of Seville, Spain

P. Real real@us.es

Dept. of Applied Math., University of Seville, Spain 